\title{
Estilos diretivos maternos apresentados a meninos e meninas
}

\author{
Fabíola de Sousa Braz Aquino \\ Universidade Estadual da Paraíba \\ Centro Universitário de João Pessoa \\ Nádia Maria Ribeiro Salomão \\ Universidade Federal da Paraíba
}

\begin{abstract}
Resumo
O presente estudo investigou a utilização de enunciados maternos diretivos, os quais podem funcionar para dirigir, controlar e manter a atenção da criança nas trocas interativas. Os enunciados diretivos podem apresentar diferentes funções nas interações e variações em seu uso dependendo de características como o gênero. Nesse estudo foram analisadas as possíveis variações no uso de diretivos maternos, em função do gênero da criança. Participaram desse estudo 16 díades mãe-criança nas idades entre 24-30 meses. As díades foram filmadas em ambiente natural durante 20 minutos. As transcrições das sessões seguiram as diretrizes do sistema computacional Child Language Data Exchange System (CHILDES). A aplicação do teste MannWhitney revelou variações no uso de diretivos maternos, sendo dirigidos mais diretivos a meninos que a meninas. Os resultados foram discutidos considerando-se o nível lingüístico infantil e os contextos nos quais os enunciado ocorreram.
\end{abstract}

Palavras-chave: interação mãe-criança; diretivos maternos; intenção comunicativa; gênero

\begin{abstract}
Maternal directive styles directed to boys and girls. The present study investigated the use of directives which can function to direct, control and keep the child's attention during the interactions. The directives utterances may show different functions in the interactions in which they are used depending on characteristics such as gender. In this study the possible variations in the use of maternal directives were analyzed by considering the child's gender. Sixteen mother-child dyads participated in this study. Children's age varied between 24-30 months. The dyads were video-recorded in natural environment for 20 minutes. The transcriptions of the sessions were carried out following the norms of the computational system Child Language Data Exchange System (CHILDES). Mann-Whitney $U$ test indicated a significant difference in the maternal speech directed to boys and girls. These results showed that the maternal directives were directed more to boys than to girls. These results were discussed considering the level of the children linguistic development and the context in which the utterances were presented.
\end{abstract}

Keywords: mother-child interaction; maternal directives; communicative intention; gender

$\mathrm{E}$ studos realizados pela perspectiva da interação social dos estudiosos da linguagem (Pine, 1992, 1994; Snow, 1989, 1977) têm enfatizado as contribuições da fala materna para o desenvolvimento cognitivo e lingüístico infantil. As pesquisas nesta área (Barnes, Gutfreund, Satterly, \& Wells, 1983; Phillips, 1973; Pine, 1992; Snow, 1977, 1989) revelaram que as mães adotam um estilo de fala peculiar ao se dirigirem às crianças pequenas que se diferencia da fala entre adultos, indicando uma adaptação da mãe às habilidades lingüísticas limitadas de crianças pequenas. Através desse "código”, ou registro de fala, a mãe parece ajustar sua linguagem ao nível de desenvolvimento cognitivo e lingüístico infantil.
Os ajustes na fala materna trazem subjacente a idéia de que as mães, ao falarem com suas crianças, estão tentando envolvê-las no diálogo, comunicar-lhes uma intenção, e ainda, que a maneira dos adultos falarem com as crianças e as circunstâncias sob as quais isso ocorre podem contribuir para o desenvolvimento de estruturas de linguagem das crianças (Gleitman, Newport, \& Gleitman, 1984). Esses ajustes na fala materna caracterizam o motherese, ou manhês, um estilo de fala que envolve enunciados curtos e simples, presença de gestos que auxiliam na comunicação e que parecem prover às crianças informações (Snow, 1977), um padrão de entonação marcado (Fernald, 1989), simplificação na forma e no conteú- 
do da fala (Ochs \& Schieffelin, 1997) e principalmente uma intenção comunicativa (Austin, 1962/1990).

Para a perspectiva da interação social os enunciados maternos podem expressar uma ampla variedade de intenções comunicativas e funções nas trocas lingüísticas. Dentre os estilos de fala materna investigados quanto a sua função nas interações pode-se mencionar os diretivos maternos, que surgem desde as primeiras interações verbais e carregam consigo uma intenção mais explícita nos diálogos (Barret, 1989; Bock \& Hornsby, 1981; Ervin-Tripp \& Gordon, 1984).

Nos primeiros anos de vida, a criança ainda não possui as informações necessárias acerca de como e quando pode fazer uso de determinados atos de fala, e de que maneira a sua estrutura sintática poderia ser utilizada para expressar uma série de intenções comunicativas (Belinger, 1979). Essa pouca habilidade cognitiva e lingüística das crianças para o manejo da linguagem em seu contexto, leva o adulto a produzir um tipo de enunciado que possui não só uma estrutura como também uma intenção diretiva claramente interpretável pela criança.

Pesquisas (Akhtar, Dunham, \& Dunham, 1991; Barret, 1989; Ervin-Tripp, 1977) realizadas acerca desse tipo de estilo comunicativo assinalam que esses enunciados de caráter diretivo, percebidos com relativa freqüência nos diálogos mãecriança desde a gênese da linguagem infantil, sofrem uma série de mudanças em seu conteúdo, à medida que a criança desenvolve sua competência lingüística.

Conforme Barret (1989), geralmente esses enunciados expressam um comando ou ordem, por parte dos pais, dirigido à criança não só para obter a atenção da mesma sob um determinado objeto, como também para enfatizar algo que foi solicitado a esta pelos pais. Com o avanço dessa competência, a criança vai diferenciando uma variedade de formas diretivas que vão de comandos simples evidentes até atos de fala mais sutis, que requerem maior inferência baseada em um conhecimento, o qual propicia a identificação de um determinado enunciado como diretivo.

$\mathrm{Na}$ visão desse autor, quando as crianças começam a adquirir sua linguagem, elas se deparam com enunciados diretivos que englobam a emissão de ordens, pedidos de objetos, obtenção de atenção e direção da atenção do outro para objetos do ambiente. As palavras olhe, vá e venha cá, por exemplo, podem ser interpretadas como servindo a funções diretivas.

A classificação de enunciados diretivos interrogativos e imperativos foi estudada por Bock e Hornsby (1981). Conforme esses autores, esses tipos de diretivos diferem apenas na sua função comunicativa. Para Searle (1995), através de sinais lingüísticos e/ou não-lingüísticos, como os gestos, as pessoas podem comunicar a intenção de uma solicitação, um comando, um compromisso ou outras comunicações convencionais. Mesmo aparentando uma intenção comunicativa diferente, esses dois tipos de diretivos possuem o mesmo sentido, a saber: obter do ouvinte a realização de uma ação desejada.

Conforme Bock e Hornsby (1981), as solicitações diretivas formuladas através de uma pergunta são geralmente percebi- das como um estilo de fala mais polido que o comando diretivo explícito. A segunda diferença apontada por esses autores entre os diretivos e as solicitações foi a forma sintática escolhida para os diretivos.

As investigações realizadas acerca da diretividade na fala materna dirigida à criança apontam para a existência de posturas teoricamente divergentes, relacionadas ao papel dos diretivos no desenvolvimento lingüístico infantil (Barnes et al., 1983; Tomasello \& Farrar, 1986; Salomão \& Conti-Ramsden, 1994).

Pine (1992) situa a origem de tais divergências no estudo realizado por Nelson, no início da década de 70, em que esta autora defende uma visão essencialmente negativa dos diretivos, equiparando-os à insensibilidade e intrusão maternas, quando de seu uso nas interações lingüísticas.

Essa idéia foi corroborada por estudos (Harris, Jones, \& Brookes, 1986; Tomasello \& Todd, 1983) os quais verificaram uma relação negativa entre as formas de diretividade materna e algumas medidas no avanço lingüístico infantil. Em contrapartida, estudos (Barnes et al., 1983; Furrow, Nelson \& Benedict, 1979) verificaram efeitos positivos da diretividade materna para o desenvolvimento da fala infantil quando as crianças estavam em estágios iniciais de sua linguagem.

Furrow et al. (1979) realizaram um estudo para avaliar os efeitos que teria a fala materna dirigida à criança para o desenvolvimento lingüístico infantil. Nesse estudo, procurouse verificar as correlações entre esse estilo de fala e o desenvolvimento da linguagem em crianças com mesma idade e estágio lingüístico. Foi verificado, através dessa pesquisa, que o uso de imperativos maternos foi positivamente relacionado às três principais medidas da linguagem infantil: a extensão média dos enunciados (MLU; $r=0,88$ ), o uso de verbos por enunciados $(r=0,91)$ e os nomes nas frases por enunciado $(r=0,76)$.

Salomão e Conti-Ramsden (1994) realizaram uma investigação comparando a fala materna dirigida à criança com distúrbio específico na linguagem e seus irmãos mais novos que apresentavam um desenvolvimento normal e estavam num mesmo estágio lingüístico, medido através do MLU. Nesse estudo, os enunciados maternos especificamente abordados foram: as solicitações, os diretivos, feedbacks, e os comportamentos comunicativos não-verbais. A ocorrência de tais enunciados pôde ser verificada durante observações de uma situação de brinquedo.

De acordo com essas autoras, verificar as semelhanças e variações da fala materna dirigida às crianças com desenvolvimento normal da linguagem e com distúrbio específico de linguagem, possibilita esclarecer quais aspectos da interação mãe-criança estão relacionados ao estilo materno e quais os aspectos que podem ser influenciados pelo prejuízo da linguagem da criança.

No que se refere aos enunciados maternos, os resultados obtidos indicaram que as mães usaram significativamente mais enunciados quando interagiam com as crianças mais novas que apresentavam um desenvolvimento normal da linguagem. Não foi verificada uma diferença significativa no número de solicitações recebidas pelos dois grupos. 
Quanto aos enunciados diretivos, observou-se que as mães utilizaram mais esse tipo de enunciado quando se dirigiram às crianças com desenvolvimento normal da linguagem $\mathrm{e}$ que essas apresentaram mais instruções imprecisas aos seus filhos, ou seja, enunciam instruções sem nomear o objeto como, por exemplo, "coloque este aqui”, "pegue aquilo ali".

Ademais, não foi observada nos dois grupos uma diferença significativa no uso pelas mães de comportamentos comunicativos não-verbais. No que diz respeito aos enunciados das crianças, não foi observada uma diferença significativa no número de respostas verbais e não-verbais adequadas entre os dois grupos.

Percebe-se que os resultados desse estudo vão na direção oposta daqueles encontrados por Conti-Ramsden (1990), já que, nessa investigação, as crianças com distúrbio específico de linguagem receberam significativamente menos diretivos que seus irmãos mais novos. Uma das explicações apresentadas para esses resultados é que as crianças com desenvolvimento normal, sendo mais novas, podem ter requerido da mãe mais comportamentos de controle e enunciados para obter a atenção desse grupo.

De acordo com Salomão e Conti-Ramsden (1994), possivelmente fatores como a idade e o nível lingüístico levam à produção de diferentes resultados. Além disso, a questão dos diretivos ainda é metodologicamente complexa no que tange à sua definição, pois diferentes pesquisadores (Barnes et al., 1983; Tomasello \& Farrar, 1986;) têm utilizado definições diferenciadas, sugerindo ainda que os diretivos podem ter efeitos diferentes no desenvolvimento da linguagem da criança.

Autores como Pine (1992) sustentam que as divergências acerca do real papel dos enunciados diretivos podem decorrer dos tipos de conceitos e distinções que têm sido propostas para caracterizar as variações encontradas nos estilos interacionais. Esse autor aponta para o erro em polarizar a função dos enunciados diretivos como favoráveis ou desfavoráveis ao avanço do vocabulário das crianças. Conforme Pine (1992), esse tipo de classificação, assim como aquela que relaciona a diretividade materna à insensibilidade, representa uma análise ainda simplificada do papel dos diretivos.

Ademais, os estudos nessa área assinalam que o modo através do qual os enunciados diretivos surgem durante as interações pode variar em função de características individuais da criança tais como seu nível de desenvolvimento cognitivo e lingüístico, sua faixa etária e as diferenças entre as crianças no que se refere a seus estilos ou estratégias para se inserirem no sistema lingüístico (Hampson \& Nelson, 1993).

Além disso, o gênero da criança tem sido apontado como um dos possíveis responsáveis pelas variações em contextos interativos mãe-criança. A partir de uma revisão da literatura, percebe-se que as pesquisas relacionadas ao gênero parecem centrar suas questões na aquisição de papéis sexualmente apropriados (Fagot \& Leinbach, 1989; Smith \& Daglish, 1977), nas diferenças entre os pais no que se refere ao tratamento e práticas educativas de meninos e meninas
(De Francisco, 1992; Fagot, 1984), e no quanto a socialização baseada em papéis sexuais pode modificar-se em função da idade das crianças (Fagot \& Hagan, 1991).

Em relação às variações em contextos interativos mãecriança, são poucos os estudos referentes aos estilos de fala materna dirigidos a meninos e meninas. Ely e Gleason (1997) verificaram que, com o desenvolvimento da linguagem das crianças, os pais lançam mão de diretivos lingüísticos explícitos como proibições, para controlar o comportamento infantil. Masur e Gleason (1980) demonstraram que uma maior produção lexical e complexidade sintática na fala da mãe foi associada com uma maior produção lexical tanto de meninos quanto de meninas, embora, em média, as meninas tenham a tendência de acelerar mais rapidamente o vocabulário que os meninos.

Pesquisa realizada por Huttenlocher, Haight, Seltzer e Lyons (1991) revelou que algumas das primeiras diferenças entre os gêneros, no que se refere ao crescimento do vocabulário, apontam variações maturacionais na capacidade lingüística de meninos e meninas. Huttenlocher e colaboradores (1991) investigaram o papel da exposição da linguagem na aquisição do vocabulário de meninos e meninas na idade entre 14 e 26 meses. Esses autores procuraram verificar se existiam diferenças entre os gêneros em relação ao crescimento do vocabulário e se essas diferenças ocorriam em função da exposição da fala materna.

Os resultados demonstraram que, embora a quantidade média de fala materna tenha sido um pouco mais alta para as mães de meninas, a diferença observada não foi significativa. Além disso, observou-se que as diferenças de gênero em relação à produção lingüística não refletem simplesmente diferenças em quão falante cada menino ou menina seja, mas diferenças reais em seu nível de vocabulário. Conforme esses autores, as diferenças de gênero no desenvolvimento inicial do vocabulário parecem indicar primeiro uma diferença na capacidade das crianças, e não apenas nas respostas diferenciais de mães a seus filhos e filhas.

Uma pesquisa realizada por Fagot e Hagan (1991) para verificar as reações dos pais aos comportamentos das crianças, demonstrou que aos 18 meses de idade, os meninos recebiam mais feedback negativo nas tentativas de comunicação que as meninas. Inversamente, as meninas recebiam mais feedback positivo do que os meninos por fazerem tentativas de participar do diálogo. Observou-se também nesse estudo que durante as interações, as meninas recebiam mais incentivos das mães para falar do que os meninos, e que, quando as crianças atingiram os cinco anos de idade, essas diferenças nas trocas comunicativas em função do gênero não foram mais observadas.

A análise dos aspectos sócio-comunicativos da fala materna e das funções que enunciados tais como os diretivos podem desempenhar na aquisição lingüística infantil pode contribuir para uma melhor caracterização dos estilos interativos das díades mãe-criança. Nesta direção, enfatizase a relevância dessa análise considerando o número reduzido de estudos realizados com crianças brasileiras sobre as 
possíveis variações nos estilos lingüísticos maternos em função do gênero da criança.

Neste sentido, buscou-se verificar no presente estudo os estilos comunicativos maternos dirigidos a meninos e meninas, num contexto de brinquedo livre. Observou-se especificamente como se apresentaram os enunciados diretivos maternos, em função dos comportamentos comunicativos verbais e não-verbais apresentados por meninos e meninas.

\section{Método}

\section{Participantes}

Participaram desse estudo, 16 díades mãe-criança, pertencentes a famílias de classe social média da cidade de João Pessoa, Paraíba. As crianças estavam na faixa etária entre 2430 meses de idade, distribuídas igualmente quanto ao gênero. Foram utilizados como parâmetro para a variável classe social, o nível de instrução da mãe (a partir do Ensino Médio completo) e a renda familiar (de 15 a 20 salários mínimos). As mães possuíam a idade superior a 20 anos, eram casadas, trabalhavam apenas um período do dia, e tinham no máximo dois filhos, além da criança que participou do estudo.

\section{Instrumentos e situação}

Para registrar os estilos de fala materna dirigidos à criança foram realizadas gravações em vídeo das interações estabelecidas entre a mãe e a criança, num contexto de brincadeira livre. Essa situação constitui um exemplo de um contexto de troca que ocorre comumente entre a mãe e a criança.

\section{Procedimentos para a coleta dos dados}

As observações foram realizadas em ambiente natural, no horário de preferência das mães. O primeiro contato com as mães, feito por telefone, teve como propósito explicar o objetivo da pesquisa, certificar-se da participação da díade no estudo e marcar uma visita em seu ambiente. Na primeira visita foi realizada a entrevista com a mãe, e, após realizada a entrevista, a pesquisadora marcou com a mãe uma segunda visita para que fosse realizada a filmagem da atividade de brinquedo livre. Na sessão de observação, a única instrução dada pela pesquisadora antes da filmagem foi a de que a mãe brincasse com seu(sua) filho(a) da maneira que costuma brincar habitualmente. Durante as gravações apenas estiveram presentes a mãe, a criança e a pesquisadora.

Após realizados todos os registros da situação de brinquedo livre, foi iniciado o processo de transcrição das sessões de observação. Essas sessões tiveram em média, vinte minutos, sendo apenas transcritos e analisados dez minutos de cada uma delas, conservando-se a forma exata das emissões verbais e não-verbais da mãe e da criança.

\section{Sistema de categorias}

Para analisar os estilos de fala materna e a participação da criança no diálogo, foram elaboradas categorias, formuladas a partir dos objetivos da pesquisa, de uma sessão de observação em ambiente natural e de dados da literatura. Es- sas categorias foram complementadas a partir de dados obtidos nas sessões de observação deste estudo, sofrendo modificações até que a codificação de todos os protocolos fosse iniciada. As verbalizações das mães e das crianças, assim como os seus comportamentos comunicativos nãoverbais, podem desempenhar diferentes funções no discurso (Van Kleeck, Maxwell, \& Gunter, 1985). Entretanto, a análise da fala materna no presente estudo baseou-se em classificações que foram mutuamente exclusivas: cada um dos enunciados era classificado em apenas uma categoria, ou seja, aquela que o pesquisador considerou como desempenhando a função principal.

As categorias de fala materna e infantil analisadas foram definidas com base nos estudos realizado por Conti-Ramsden (1990), Akhtar e colaboradores (1991); Pine (1992) e Salomão (1996). Essas categorias estão dispostas no Apêndice.

\section{Procedimento para a análise dos dados}

Após a etapa de transcrição das sessões ser concluída, esses dados foram dispostos no computador, seguindo as normas do Child Language Data Exchange System (CHILDES), um sistema computacional composto de três instrumentos que visam facilitar a troca de dados entre diferentes pesquisadores, aumentar a fidedignidade das transcrições e automatizar o processo de análise de dados (Sokolov \& Snow, 1994). O sistema CHILDES atinge esses três objetivos através de três diferentes instrumentos que estão integrados: o CHAT (Codes for Human Analysis of Transcripts) que corresponde ao sistema padronizado de transcrição; o CLAN (Computerised Language Analysis), no qual os programas são desenhados para desenvolver análises dos dados transcritos tais como contagem de freqüência, procura de palavras, análise interacional, cálculo de MLU, mudanças no texto, dentre outros; e o CHILDES, que é um banco de dados em que é possível ter acesso a um grande número de projetos de pesquisas e a uma enorme variedade de dados lingüísticos.

A inserção dos dados transcritos no programa CHILDES foi realizada considerando-se os enunciados da mãe e da criança separados em unidades verbais, levando em conta três critérios, a saber: a troca de interlocutor, a pausa com intervalo de tempo maior que dois segundos para separar sucessivos enunciados de um mesmo locutor, e verbalizações que, mesmo não separadas por este intervalo, apresentem um conteúdo semântico diferente da emissão que a precedeu (Salomão, 1996). Posteriormente iniciou-se a fase de codificação dos enunciados, que foi realizada no CLAN, quando foram efetivados a contagem de freqüência das categorias e o cálculo da extensão média dos enunciados (MLU) da mãe e da criança.

O índice de fidedignidade do registro das categorias de atos de fala da mãe e da criança foi calculado através da fórmula IF $=(\Sigma \mathrm{A} / \Sigma(\mathrm{A}+\mathrm{D})$. 100). Para verificar a fidedignidade dessa codificação, $20 \%$ do material foi analisado por outro codificador, e, após esse processo, as porcentagens de concordância deste segundo codificador foram comparadas 
com as do codificador original. A partir dessa comparação, obteve-se $91,5 \%$ de concordância das categorias de fala materna e infantil.

\section{Resultados}

Os resultados das comparações entre os estilos de fala materna utilizados por mães de meninos e meninas, e dos comportamentos comunicativos infantis, foram extraídos a partir do cálculo de proporções das emissões verbais e não verbais das mães e crianças de ambos os grupos; esse cálculo possibilitou a descrição de dados referentes às diferenças entre os grupos.

A proporção foi calculada pela frequiência total de cada tipo de enunciado materno dividido pelo número total das categorias de fala materna dirigidas à criança, e multiplicado por cem. Também foi realizado o cálculo de proporções com os comportamentos comunicativos do grupo das crianças, através do mesmo processo. Conforme Hoff-Ginsberg (1992a, 1992b), esse cálculo possibilita captar diferentes aspectos da fala materna, e aspectos do input que auxiliam a aprendizagem da linguagem infantil. Após terem sido realizados esses cálculos, foi aplicado o teste de Mann-Whitney $(U)$ para verificar se havia diferença significativa entre as proporções de comportamentos verbais e não-verbais das mães e crianças em ambos os grupos.

A Tabela 1 exibe as proporções gerais de diretivos maternos apresentados a meninos e meninas. Verificou-se uma diferença significativa nos diretivos maternos dirigidos ao grupo de meninos, quando comparado com o grupo de meninas $(p<0,05)$. A Tabela 1 demonstra ainda os tipos de diretivos maternos dirigidos a meninos e meninas. Como pode-se verificar, não foi observada diferença significativa nas médias proporcionais dos tipos de diretivos maternos em relação aos dois grupos, embora tenha sido observada uma maior proporção de diretivos de instrução dirigida ao grupo de meninas.

Uma análise detalhada dos protocolos de observação revelou que, geralmente, os contextos específicos nos quais os diretivos ocorreram em ambos os grupos foram: (a) aqueles em que as mães davam instrução para que a criança realizasse algo; (b) aqueles em que a mãe chamava a atenção da criança quando esta não estava atenta a ela; (c) quando as mães impediam fisicamente um comportamento da criança; e (d) quando a mãe pedia que a criança repetisse algo, sendo os dois primeiros contextos os mais freqüentemente utilizados pelas mães durante as interações.

\section{Discussão}

Como já mencionado, a literatura nessa área tem investigado a função comunicativa de uma série de estilos de fala materna, dentre eles os diretivos. Esse tipo de enunciado tem sido questionado quanto às suas possíveis implicações para o desenvolvimento da linguagem infantil. O presente trabalho teve como objetivo verificar se havia variações nos estilos comunicativos maternos, marcadamente nos diretivos, dirigidos a meninos e meninas.

As análises dos enunciados maternos revelaram variações nos comportamentos comunicativos maternos em função do gênero da criança. Primeiramente, verificou-se que as mães de meninos emitiram significativamente mais diretivos que as mães de meninas. Esses resultados corroboram aqueles encontrados por Perlmann e Gleason (1990, citados por Ely \& Gleason, 1997), os quais encontraram em sua pesquisa que diretivos lingüísticos maternos foram mais dirigidos a meninos que a meninas.

A análise dos tipos específicos de diretivos maternos dirigidos aos dois grupos revelou que foram mais utilizados pelas mães de meninos os diretivos de atenção, enquanto os diretivos de instrução foram mais utilizados pelas mães de meninas. Mesmo não tendo sido verificada uma diferença significativa nas proporções desses dois tipos de enunciados em relação aos dois grupos, observou-se que os contextos nos quais eles ocorreram diferiram quanto à sua intenção comunicativa durante as interações entre as díades dos dois grupos.

Os diretivos de atenção foram utilizados especificamente nos momentos em que a criança não estava atenta a uma fala ou objeto ao qual a mãe se referia, enquanto os diretivos de instrução foram utilizados basicamente para controlar o comportamento das crianças através de comandos ou solicitações explícitas, que geralmente demonstravam a habilidade da criança com objetos e conceitos.

Uma outra leitura destes dados deve considerar ainda que uma maior utilização de diretivos de atenção dirigido aos meninos pode indicar que houve um menor envolvimento ou atenção destes nas atividades propostas pelas mães. Já nas

Tabela 1

Diretivos maternos apresentados a meninos e meninas $(N=16)$

\begin{tabular}{lrrr}
\hline \multirow{2}{*}{ Diretivos maternos } & \multicolumn{2}{c}{ Média de postos } & \multirow{2}{*}{ Teste U } \\
\cline { 2 - 3 } & Meninos Meninas & \\
\hline Diretivo geral & 11,25 & 5,75 & $10,0^{*}$ \\
Diretivos de instrução & 6,94 & 10,06 & 19,5 \\
Diretivos de atenção & 10,31 & 6,69 & 17,5 \\
Diretivos de controle de comportamento & 8,50 & 8,50 & 32,0 \\
Diretivo de repetição & 8,50 & 8,50 & 32,0 \\
\hline
\end{tabular}

$* p<0,05$ 
díades mãe-menina foi observada uma menor proporção de diretivos de atenção, o que pode indicar que as meninas se engajaram mais nas interações, durante a situação de brincadeira livre.

Desta feita, esses dados podem indicar que os diretivos maternos de atenção e instrução, mais freqüentes nesse estudo, podem ter veiculado diferentes intenções comunicativas e não apenas a intenção de controlar e inibir o comportamento da criança. Essa idéia ganha apoio na afirmação de Pine (1992), que considera inadequada a tendência da literatura em tratar diferentes formas de diretividade materna como se fossem essencialmente equivalentes, ou seja, como se todos os tipos de diretivos maternos carregassem em si a mesma função comunicativa.

A análise do estilo de fala diretiva, neste estudo, revelou que uma investigação criteriosa desse tipo de enunciado deve considerar as diversas funções dos Diretivos nos diálogos. As estratégias metodológicas utilizadas para sua análise devem estar bem definidas para que o uso dos diretivos maternos não seja interpretado de forma polarizada, como argumentado por Pine (1992). Já que esses enunciados podem apresentar diversas funções nos diálogos, sua análise deve considerar os contextos em que são emitidos, o nível de desenvolvimento lingüístico infantil, e as maneiras através das quais os diretivos são apresentados às crianças.

Pôde-se verificar que características infantis como o gênero, podem provocar estilos interativos peculiares não apenas pelo fato das crianças serem meninos ou meninas, mas pelo tipo de relação que estabelecem com suas mães desde uma idade bem precoce. É importante lembrar que a maneira através da qual a mãe usa esse tipo de enunciado nos contextos lingüísticos modifica-se à medida que a criança vai estabelecendo com a mãe padrões de interação mais sofisticados e complexos. Além disso, os contextos e atividades nos dois grupos variaram, o que pode ter contribuído para que fossem observadas diferenças no uso dos diretivos maternos.

Ademais, destaca-se a relevância de estudos que abordem os estilos comunicativos maternos numa abordagem longitudinal enfocando as mudanças que ocorrem nesses estilos em função de características como a idade e o nível lingüístico infantil.

\section{Agradecimento}

As autoras agradecem à CAPES, pelo financiamento da presente pesquisa.

\section{Referências}

Akhtar, N., Dunham, F., \& Dunham, P. J. (1991). Directive interactions and early vocabulary development: the role of joint attentional focus. Journal of Child Language, 18, 41-49.

Austin, J. L. (1990). Quando dizer é fazer: palavras e ação. Porto alegre: Artes Médicas. (Texto original publicado em 1962)

Barnes, S., Gutfreund, M., Satterly, D., \& Wells, G. (1983). Characteristics of adult speech which predict children's language development. Journal of Child Language, 10, 65-84.
Barret, M. (1989). Early language development. In A. Slater \& G. Bremner (Orgs.), Infant development (pp. 211-241). Hillsdale, New Jersey: Lawrence Erlbaum.

Bellinger, D. (1979). Changes in the explicitness of mothers' directives as children age. Journal of Child Language, 6, 443-445.

Bock, J. K., \& Hornsby, M. E. (1981). The development of directives: how children ask and tell. Journal of Child Language, 8, 151-163.

Conti-Ramsden, G. (1990). Maternal recasts and other contingent replies to language-impaired children. Journal of Speech and Hearing Disorders, 55, 262-274.

DeFrancisco, V. L. (1992). Ethnography and gender: learning to talk like girl and boys. Topics in Language Disorders, 12(3), 40-53.

Ely, R., \& Gleason, J. B. (1997). Socialização em diferentes contextos. In P. Fletcher \& B. Macwinney (Orgs.), Compêndio da linguagem da criança (pp. 209-224). Porto Alegre: Artes Médicas.

Ervin-Tripp, S. (1977). Wait for me, roller skate! In S. Ervin-Tripp \& MitchellKernan (Orgs.), Language, thought and culture - advances in the study of cognition (pp. 165-188). Nova York: Academic.

Ervin-Tripp, S., \& Gordon, D. (1984). The development of request. In L. Schiefelbusch \& J. Pickar (Orgs.), The acquisition of communication competence (pp. 61-95). Baltimore: University Park Press.

Fagot, B. (1984). The child's expectations of differences in adult male and female interaction. Sex Roles, 11, 593-600.

Fagot, B., \& Hagan, R. (1991). Observations of parent reactions to sex-stereotyped behaviors: age and sex effects. Child Development, 62, 617-628.

Fagot, B., \& Leinbach, M. D. (1989). The young child's gender schema: environmental input, internal organization. Child Development, 60, 663-672.

Fernald, A. (1989). Intonation and communicative intent in mothers' speech to infant: is the melody the message? Child Development, 60, 1497-1510.

Furrow, D., Nelson, K., \& Benedict, H. (1979). Mothers' speech to children and syntactic development: some simple relationships. Journal of Child Language, 6, 423-442.

Gleitman, L. R., Newport, E. L., \& Gleitman, H. (1984). The current status of the motherese hypothesis. Journal of Child Language, 11, 43-79.

Hampson, J., \& Nelson, K. (1993). The relation of maternal language to variation in rate and style of language acquisition. Journal of Child Language, 20, 313-342.

Harris, M., Jones, D., \& Brookes, S. (1986). Maternal speech and early language. In R. Crawley, R. Stevenson, \& M. Tallerman (Orgs.), Proceedings of the child language seminar (pp. 75-87). Durham, Reino Unido: University of Durham.

Hoff-Ginsberg, E. (1992a). How should frequency in input be measured? First Language, 12, 233-244.

Hoff-Ginsberg, E. (1992b). Methodological and social concerns in the study of children's language-learning environments: a reply to Pine. First Language, 12, 251-254.

Huttenlocher, J., Haight, W., Bryk, A., Seltzer, M., \& Lyons, T (1991). Early vocabulary growth: Relation to language input and gender. Development Psychology, 27(2), 236-248.

Masur, E. F., \& Gleason, J. B. (1980). Parent-child interaction and the acquisition of lexical information during play. Developmental Psychology, 16, 5, 404-409.

Ochs, E., \& Schieffelin, B. (1997). O impacto da socialização da linguagem no desenvolvimento gramatical. In P. Fletcher \& B. MacWhinney (Orgs.), Compêndio da linguagem da criança (pp. 69-84). Porto Alegre: Artes Médicas.

Phillips, J. R. (1973). Syntax and vocabulary of mothers' speech to young children: age and sex comparisons. Child Development, 44, 182-185.

Pine, J. M. (1992). Maternal style at the early one-word stage: re-evaluating the stereotype of the directive mother. First Language, 12, 169-186. 
Pine, J. M. (1994). The language of primary caregivers. In C. Gallaway \& B. Richards (Orgs.), Input and interaction in language acquisition (pp.15-37). Londres: Cambridge University Press.

Salomão, N. M. R., \& Conti-Ramsden, G. (1994). Maternal speech to their offspring: SLI children and their younger siblings. Scandinavian Journal of Logopedics and Phonology, 19, 11-17.

Salomão, N. M. R (1996). Interaction between mothers and children with specific language impairment: a longitudinal study. Tese de doutorado nãopublicada, Universidade de Manchester, Manchester, Reino Unido.

Searle, J. R. (1995). Intencionalidade. São Paulo: Martins Fontes.

Smith, P. K., \& Daglish, L. (1977). Sex differences in parental and infant behavior in the home. Child Development, 48, 1250-1254.

Snow, C. E. (1977). The development of conversation between mothers and babies. In V. Lee (Org.), Language development (pp. 235-249). Londres: The Open University.
Snow, C. E. (1989). Understanding social interaction and language acquisition; sentences are not enough. In M. H. Bornstein \& J. S. Bruner (Orgs.), Interaction in human development (pp. 83-103). Hillsdale, New Jersey: Lawrence Erlbaum.

Sokolov, J. L., \& Snow, C. E. (1994). The changing role of negative evidence in theories of language development. In C. Gallaway \& B. Richards (Orgs.), Input and interaction in language acquisition (pp.38-55). Londres: Cambridge University Press.

Tomasello, M., \& Farrar, M. J. (1986). Joint attention and early language. Child Development, 57, 1454-1463.

Tomasello, M., \& Todd, J. (1983). Joint attention and lexical acquisition style. First Language, 4, 197-212.

Van-Kleek, A., Maxwell, E., \& Gunter, P. (1985). A methodological study of elocutionary coding in adult-child interaction. Journal of Pragmatics, 9, 659-681.

Fabíola de Sousa Braz Aquino, doutoranda pelo Doutorado Integrado UFPB-UFRN em Psicologia Social, é professora na Universidade Estadual da Paraíba e no Centro Universitário de João Pessoa.

Nádia Maria Ribeiro Salomão, doutora em Psicologia pela UniverSidade de Manchester (Reino Unido), é professora no Departamento de Psicologia da Universidade Federal da Paraíba. Endereço para correspondência: Departamento de Psicologia; Universidade Federal da Paraíba; João Pessoa, PB; CEP: 58.059-900. Email: nmrs@uol.com.br 


\section{Apêndice}

\section{Estilos diretivos de fala materna}

Diretivos podem ser interpretados como um comando ou ordem, possuindo um componente imperativo interpretável, que dirige o comportamento ou verbalizações da criança (Akhtar, Dunham, \& Dunham, 1991).

(a) Diretivo de instrução (DIR:INS): a mãe verbaliza explicitamente o que deseja da criança, usualmente relacionado aos brinquedos que estão utilizando. Ex: Mãe: “coloque o carrinho na caixa” (olha para a criança).

(b) Diretivos de atenção (DIR:AT): a mãe chama a atenção da criança, usualmente pelo seu nome, pedindo que ela se aproxime ou olhe para algo. Ex: Mãe: “ali ó, o mickey!”. (aponta para o brinquedo).

(c) Diretivos de repetição (DIR:REP): a mãe pede à criança para repetir a palavra ou sentença dela. Ex: Mãe: “diga assim, dois" (mostra para a criança o número usando os dedos das mãos).

(d) Diretivo de controle do comportamento (DIR:CC): a mãe expressa uma objeção ao comportamento da criança. Ex: Mãe: "não pegue nisso!" (pega no braço da criança impedindo-a de pegar o objeto).

\section{Comportamentos comunicativos da criança}

1. Fala espontânea (FES): qualquer comportamento verbal da criança que não é precedido por uma pergunta, não é uma imitação ou repetição de um enunciado prévio da mãe (Salomão \& Conti-Ramsden, 1994). Ex: Criança: "vô fazer um pêxinho aqui...” (desenha no caderno).

2. Respostas da criança aos enunciados maternos:

(a) Resposta verbal adequada (RVA): a criança responde a um enunciado prévio da mãe corretamente (Salomão \& ContiRamsden, 1994). Ex: Mãe: “que cor é esse círculo?” (aponta para o desenho). Criança: “vermelho” (olha para o desenho); Mãe: “é, o vermelho!”

(b) Resposta verbal inadequada (RVI): a resposta da criança a um enunciado prévio da mãe não é correta (Salomão \& ContiRamsden, 1994). Ex: Mãe: “quem vem depois do dois?” (aponta para o brinquedo); Criança: “...cinco” (olha pra mãe). Mãe: "não, três!".

(c) Resposta não-verbal adequada (RNVA): resposta não-verbal da criança a um enunciado prévio da mãe é correta ou apropriada (Salomão \& Conti-Ramsden, 1994). Ex: Mãe: “coloque a roupinha da boneca...!” (a criança veste a roupa da boneca de forma correta).

(d) Resposta não-verbal inadequada (RNVI): a criança dá uma resposta não-verbal incorreta (Salomão \& Conti-Ramsden, 1994). Ex: Mãe: “encaixe a pecinha no reloginho”; Criança: 0 (encaixa a peça no lugar errado).

3. Não resposta (NR): a criança não responde à pergunta feita pela mãe (Salomão \& Conti-Ramsden, 1994). Ex: Mãe: “onde está seu carro vermelho?”; Criança: 0 (olha para os brinquedos dispostos no chão).

4. Repetição dos enunciados maternos:

(a) Repetição do enunciado da mãe (REM): a criança usa a mesma forma utilizada pela mãe (Salomão \& Conti-Ramsden, 1994). Ex: Mãe: "vamo brincar de trenzinho?” (olha para a criança); Criança: "brincar de trenzinho...”

5. Auto-repetição (AUTREP): a criança repete a mesma palavra ou enunciado várias vezes. Ex: Criança: "o carrinho é meu, o carrinho é meu...” (pega um carrinho no chão)

6. Ininteligível: um enunciado em que é impossível de se compreender o que o falante está dizendo (Salomão \& Conti-Ramsden, 1994). 